PROCEEDINGS OF THE

AMERICAN MATHEMATICAL SOCIETY

Volume 135, Number 11, November 2007, Pages 3515-3520

S 0002-9939(07)08890-9

Article electronically published on August 15, 2007

\title{
A SHARP RESULT ON $m$-COVERS
}

\author{
HAO PAN AND ZHI-WEI SUN
}

(Communicated by Wen-Ching Winnie Li)

\begin{abstract}
Let $A=\left\{a_{s}+n_{s} \mathbb{Z}\right\}_{s=1}^{k}$ be a finite system of residue classes which forms an $m$-cover of $\mathbb{Z}$ (i.e., every integer belongs to at least $m$ members of $A)$. In this paper we show the following sharp result: For any positive integers $m_{1}, \ldots, m_{k}$ and $\theta \in[0,1)$, if there is $I \subseteq\{1, \ldots, k\}$ such that the fractional part of $\sum_{s \in I} m_{s} / n_{s}$ is $\theta$, then there are at least $2^{m}$ such subsets of $\{1, \ldots, k\}$. This extends an earlier result of M. Z. Zhang and an extension by Z. W. Sun. Also, we generalize the above result to $m$-covers of the integral ring of any algebraic number field with a power integral basis.
\end{abstract}

\section{INTRODUCTION}

For an integer $a$ and a positive integer $n$, we simply let $a(n)$ represent the set $a+n \mathbb{Z}=\{x \in \mathbb{Z}: x \equiv a(\bmod n)\}$. Following Sun [S95, S96] we call a finite system

$$
A=\left\{a_{s}\left(n_{s}\right)\right\}_{s=1}^{k}
$$

of such sets an $m$-cover of $\mathbb{Z}$ (where $m \in\{1,2,3, \ldots\}$ ) if every integer lies in at least $m$ members of (1.1). We use the term cover (or covering system) instead of 1-cover. For problems and results in this area, the reader may consult [G04, pp. 383-390], [PS] and [S05]. P. Erdős [E97] once said: "Perhaps my favorite problem of all concerns covering systems."

Example 1.1. For each integer $m \geqslant 1$, there is an $m$-cover of $\mathbb{Z}$ that is not the union of two covers of $\mathbb{Z}$. To wit, we let $p_{1}, \ldots, p_{r}$ be distinct primes with $r \geqslant 2 m-1$, and set $N=p_{1} \cdots p_{r}$. Clearly $A_{*}=\left\{\prod_{s \in I} p_{s}(N)\right\}_{I \subseteq\{1, \ldots, r\},|I| \geqslant m}$ does not cover any integer relatively prime to $N$. Let $a_{1}, \ldots, a_{n}$ be the list of those integers in $\{0,1, \ldots, N-1\}$ not covered by $A_{*}$ with each occurring exactly $m$ times. If $x \in \mathbb{Z}$ is covered by $A_{*}$, then $x \in \bigcap_{s \in I} 0\left(p_{s}\right)$ for some $I \subseteq\{1, \ldots, r\}$ with $|I| \geqslant m$. Therefore

$$
A_{0}=\left\{0\left(p_{1}\right), \ldots, 0\left(p_{r}\right), a_{1}(N), \ldots, a_{n}(N)\right\}
$$

forms an $m$-cover of $\mathbb{Z}$. Suppose that $I_{1} \cup I_{2}=\{1, \ldots, r\}, J_{1} \cup J_{2}=\{1, \ldots, n\}$ and $I_{1} \cap I_{2}=J_{1} \cap J_{2}=\emptyset$. For $i=1,2$ let $A_{i}$ be the system consisting of those $0\left(p_{s}\right)$ with $s \in I_{i}$ and those $a_{t}(N)$ with $t \in J_{i}$. We claim that $A_{1}$ or $A_{2}$ is not a cover of $\mathbb{Z}$. Without loss of generality, let us assume that $\left|I_{1}\right| \leqslant\left|I_{2}\right|$. Since $2\left|I_{2}\right| \geqslant\left|I_{1}\right|+\left|I_{2}\right|>2(m-1)$, we have $\left|I_{2}\right| \geqslant m$ and hence $\prod_{s \in I_{2}} p_{s}$ is covered

Received by the editors January 3, 2006 and, in revised form, June 3, 2006 and August 25, 2006

2000 Mathematics Subject Classification. Primary 11B25; Secondary 11B75, 11D68, 11R04.

The second author is responsible for communications and is supported by the National Science Fund for Distinguished Young Scholars (No. 10425103) in China.

(C)2007 American Mathematical Society Reverts to public domain 28 years from publication 
by $A_{*}$. Therefore $\prod_{s \in I_{2}} p_{s} \notin \bigcup_{t=1}^{n} a_{t}(N)$. Clearly $\prod_{s \in I_{2}} p_{s}$ is not covered by $\left\{0\left(p_{s}\right)\right\}_{s \in I_{1}}$ either. Thus $A_{1}$ does not form a cover of $\mathbb{Z}$.

By means of the Riemann zeta function, in $1989 \mathrm{M}$. Z. Zhang [Z89] proved that if (1.1) forms a cover of $\mathbb{Z}$, then $\sum_{s \in I} 1 / n_{s}$ is a positive integer for some $I \subseteq\{1, \ldots, k\}$.

Let $m_{1}, \ldots, m_{k}$ be any positive integers. If (1.1) is a cover of $\mathbb{Z}$, then $\left\{a_{s}+\right.$ $\left.\left(n_{s} / m_{s}\right) \mathbb{Z}\right\}_{s=1}^{k}$ is also a cover of $\mathbb{Z}$, and hence Theorem 2 of [S95] indicates that for any $J \subseteq\{1, \ldots, k\}$ there is an $I \subseteq\{1, \ldots, k\}$ with $I \neq J$ such that $\left\{\sum_{s \in I} m_{s} / n_{s}\right\}=$ $\left\{\sum_{s \in J} m_{s} / n_{s}\right\}$, where $\{\alpha\}$ denotes the fractional part of a real number $\alpha$. When $J=\emptyset$ and $m_{1}=\cdots=m_{k}=1$, this yields Zhang's result. In 1999 Z. W. Sun S99] proved further that if (1.1) forms an $m$-cover of $\mathbb{Z}$, then for any $J \subseteq\{1, \ldots, k\}$ we have

$$
\mid\left\{I \subseteq\{1, \ldots, k\}: I \neq J \text { and }\left\{\sum_{s \in I} \frac{m_{s}}{n_{s}}\right\}=\left\{\sum_{s \in J} \frac{m_{s}}{n_{s}}\right\}\right\} \mid \geqslant m .
$$

In this paper we will show the following sharp result.

Theorem 1.1. Let (1.1) be an $m$-cover of $\mathbb{Z}$, and let $m_{1}, \ldots, m_{k}$ be any integers. Then for any $0 \leqslant \theta<1$ the set

$$
I_{A}(\theta)=\left\{I \subseteq\{1, \ldots, k\}:\left\{\sum_{s \in I} \frac{m_{s}}{n_{s}}\right\}=\theta\right\}
$$

has at least $2^{m}$ elements if it is nonempty.

Remark 1.1. Clearly $m$ copies of $0(1)$ form an $m$-cover of $\mathbb{Z}$. This shows that the lower bound in Theorem 1.1 is best possible.

Corollary 1.1. Let (1.1) be an $m$-cover of $\mathbb{Z}$, and let $m_{1}, \ldots, m_{k}$ be any integers. Then $|S(A)| \leqslant 2^{k-m}$, where

$$
S(A)=\left\{\left\{\sum_{s \in I} \frac{m_{s}}{n_{s}}\right\}: I \subseteq\{1, \ldots, k\}\right\} .
$$

Proof. As $\left|I_{A}(\theta)\right| \geqslant 2^{m}$ for all $\theta \in S(A)$, we have

$$
|S(A)| 2^{m} \leqslant|\{I: I \subseteq\{1, \ldots, k\}\}|=2^{k}
$$

and hence $|S(A)| \leqslant 2^{k-m}$.

Remark 1.2. Sun [S95, S96] showed that if $m_{1}, \ldots, m_{k}$ are relatively prime to $n_{1}, \ldots, n_{k}$, respectively, then (1.1) forms an $m$-cover of $\mathbb{Z}$ whenever it covers $|S(A)|$ consecutive integers at least $m$ times.

Corollary 1.2. Suppose that (1.1) forms an $m$-cover of $\mathbb{Z}$ but $\left\{a_{s}\left(n_{s}\right)\right\}_{s=1}^{k-1}$ does not. If the covering function $w_{A}(x)=\left|\left\{1 \leqslant s \leqslant k: x \in a_{s}\left(n_{s}\right)\right\}\right|$ is periodic modulo $n_{k}$, then for any $r=0, \ldots, n_{k}-1$ we have

$$
\left|\left\{I \subseteq\{1, \ldots, k-1\}:\left\{\sum_{s \in I} \frac{1}{n_{s}}\right\}=\frac{r}{n_{k}}\right\}\right| \geqslant 2^{m-1} .
$$

Proof. By Theorem 1 of Sun [S07,

$$
\mid\left\{\left\lfloor\sum_{s \in I} \frac{1}{n_{s}}\right\rfloor: I \subseteq\{1, \ldots, k-1\} \text { and }\left\{\sum_{s \in I} \frac{1}{n_{s}}\right\}=\frac{r}{n_{k}}\right\} \mid \geqslant m \text {. }
$$


In particular, $\left\{\sum_{s \in I} 1 / n_{s}\right\}=r / n_{k}$ for some $I \subseteq\{1, \ldots, k-1\}$, and hence (1.4) holds in the case $m=1$. For $A_{k}=\left\{a_{s}\left(n_{s}\right)\right\}_{s=1}^{k-1}$, clearly $w_{A_{k}}(x) \geqslant m-1$ for all $x \in \mathbb{Z}$. In the case $m>1$, we obtain (1.4) by applying Theorem 1.1 to $A_{k}$ with $m_{1}=\cdots=m_{k-1}=1$ and $\theta=r / n_{k}$.

Remark 1.3. When $n_{k}$ is divisible by all the moduli $n_{1}, \ldots, n_{k}$, Corollary 1.2 was stated by the second author in [S03, Theorem 2.5]. When $w_{A}(x)=m$ for all $x \in \mathbb{Z}$, the following result stronger than (1.4) (with $r \in\left\{0, \ldots, n_{k}-1\right\}$ ) was proved in [S97:

$$
\left|\left\{I \subseteq\{1, \ldots, k-1\}: \sum_{s \in I} \frac{1}{n_{s}}=n+\frac{r}{n_{k}}\right\}\right| \geqslant\left(\begin{array}{c}
m-1 \\
n
\end{array}\right)
$$

for every $n=0, \ldots, m-1$.

For an algebraic number field $K$, let $O_{K}$ be the ring of algebraic integers in $K$. For $\alpha, \beta \in O_{K}$, we set

$$
\alpha+\beta O_{K}=\left\{\alpha+\beta \omega: \omega \in O_{K}\right\}
$$

and call it a residue class in $O_{K}$. For a finite system

$$
\mathcal{A}=\left\{\alpha_{s}+\beta_{s} O_{K}\right\}_{s=1}^{k}
$$

of such residue classes, if $\left|\left\{1 \leqslant s \leqslant k: x \in \alpha_{s}+\beta_{s} O_{K}\right\}\right| \geqslant m$ for all $x \in O_{K}$ (where $m \in\{1,2,3, \ldots\}$ ), then we call $\mathcal{A}$ an $m$-cover of $O_{K}$. Covers of the ring $\mathbb{Z}[\sqrt{-2}]=O_{\mathbb{Q}(\sqrt{-2})}$ were investigated by J. H. Jordan [J68].

An algebraic number field $K$ of degree $n$ is said to have a power integral basis if there is $\gamma \in O_{K}$ such that $1, \gamma, \ldots, \gamma^{n-1}$ form a basis of $O_{K}$ over $\mathbb{Z}$. It is well known that all quadratic fields and cyclotomic fields have power integral bases.

Here is a generalization of Theorem 1.1.

Theorem 1.2. Let $K$ be an algebraic number field with a power integral basis. Suppose that (1.5) forms an m-cover of $O_{K}$, and let $\omega_{1}, \ldots, \omega_{k} \in O_{K}$. Then, for any $\mu \in K$, the set

$$
\left\{I \subseteq\{1, \ldots, k\}: \sum_{s \in I} \frac{\omega_{s}}{\beta_{s}} \in \mu+O_{K}\right\}
$$

is empty or it has at least $2^{m}$ elements.

Remark 1.4. We conjecture that the requirement in Theorem 1.2 that $K$ has a power integral basis can be cancelled.

\section{Proof of Theorem 1.1}

Lemma 2.1. Let (1.1) be an $m$-cover of $\mathbb{Z}$. Let $m_{1}, \ldots, m_{k} \in \mathbb{Z}$ and define $S(A)$ as in (1.3). Then, for any given $\theta \in S(A)$, there exists $t \in\{1, \ldots, k\}$ such that both $\theta$ and $\left\{\theta-m_{t} / n_{t}\right\}$ lie in $S\left(A_{t}\right)$, where $A_{t}=\left\{a_{s}\left(n_{s}\right)\right\}_{1 \leqslant s \leqslant k, s \neq t}$.

Proof. Choose a maximal $J \subseteq\{1, \ldots, k\}$ such that $\left\{\sum_{s \in J} m_{s} / n_{s}\right\}=\theta$. As (1.1) is a cover of $\mathbb{Z}$, by [S95, Theorem 2] or $[\mathbf{S 9 9}$, Theorem 1(i)] there exists $I \subseteq\{1, \ldots, k\}$ for which $I \neq J$ and $\left\{\sum_{s \in I} m_{s} / n_{s}\right\}=\theta$. Note that $J \not I I$ and hence $t \in J \backslash I$ for some $1 \leqslant t \leqslant k$. Clearly $\theta=\left\{\sum_{s \in I} m_{s} / n_{s}\right\} \in S\left(A_{t}\right)$ and also $\left\{\theta-m_{t} / n_{t}\right\}=$ $\left\{\sum_{s \in J \backslash\{t\}} m_{s} / n_{s}\right\} \in S\left(A_{t}\right)$. This concludes the proof. 
Proof of Theorem 1.1. We use induction on $m$.

The $m=1$ case, as mentioned above, has been handled in [S95, S99].

Now let $m>1$ and assume that Theorem 1.1 holds for smaller positive integers. Let $\theta \in S(A)$. In light of Lemma 2.1, there is $t \in\{1, \ldots, k\}$ such that both $\theta$ and $\theta^{\prime}=\left\{\theta-m_{t} / n_{t}\right\}$ lie in $S\left(A_{t}\right)$. As $A_{t}$ forms an $(m-1)$-cover of $\mathbb{Z}$, by the induction hypothesis we have $\left|I_{A_{t}}(\theta)\right| \geqslant 2^{m-1}$ and $\left|I_{A_{t}}\left(\theta^{\prime}\right)\right| \geqslant 2^{m-1}$. Observe that

$$
I_{A}(\theta)=I_{A_{t}}(\theta) \cup\left\{I \cup\{t\}: I \in I_{A_{t}}\left(\theta^{\prime}\right)\right\} .
$$

Therefore

$$
\left|I_{A}(\theta)\right|=\left|I_{A_{t}}(\theta)\right|+\left|I_{A_{t}}\left(\theta^{\prime}\right)\right| \geqslant 2^{m-1}+2^{m-1}=2^{m} .
$$

We are done.

\section{Proof of Theorem 1.2}

At first we give a lemma on algebraic number fields with power integral bases.

Lemma 3.1. Let $K$ be an algebraic number field with a power integral basis $1, \gamma, \ldots$, $\gamma^{n-1}$. For any $\mu=\sum_{r=0}^{n-1} \mu_{r} \gamma^{r} \in K$ with $\mu_{0}, \ldots, \mu_{n-1} \in \mathbb{Q}$, we have

$$
\mu \in O_{K} \Longleftrightarrow \psi(\mu), \psi(\mu \gamma), \ldots, \psi\left(\mu \gamma^{n-1}\right) \in \mathbb{Z},
$$

where $\psi(\mu)$ denotes the last coordinate $\mu_{n-1}$ of $\mu$.

Proof. If $\mu \in O_{K}$, then $\mu, \mu \gamma, \ldots, \mu \gamma^{n-1} \in O_{K}$ and hence $\psi\left(\mu \gamma^{j}\right) \in \mathbb{Z}$ for every $j=0, \ldots, n-1$.

Now assume that $\psi\left(\mu \gamma^{j}\right) \in \mathbb{Z}$ for all $j=0, \ldots, n-1$. We want to show that $\mu \in O_{K}$ (i.e., $\mu_{0}, \ldots, \mu_{n-1} \in \mathbb{Z}$ ). Clearly $\mu_{n-1}=\psi\left(\mu \gamma^{0}\right) \in \mathbb{Z}$. If $0 \leqslant r<n-1$ and $\mu_{r+1}, \ldots, \mu_{n-1} \in \mathbb{Z}$, then

$$
\begin{aligned}
\mu_{r} & =\psi\left(\mu_{0} \gamma^{n-1-r}+\mu_{1} \gamma^{n-r}+\cdots+\mu_{r} \gamma^{n-1}\right) \\
& =\psi\left(\mu \gamma^{n-1-r}\right)-\psi\left(\mu_{r+1} \gamma^{n}+\cdots+\mu_{n-1} \gamma^{2 n-2-r}\right)
\end{aligned}
$$

and hence $\mu_{r} \in \mathbb{Z}$ since $\mu_{r+1} \gamma^{n}+\cdots+\mu_{n-1} \gamma^{2 n-2-r} \in O_{K}$. So, by induction, $\mu_{r} \in \mathbb{Z}$ for all $r=0, \ldots, n-1$. We are done.

Proof of Theorem 1.2. In the spirit of the proof of Theorem 1.1, it suffices to handle the case $m=1$. That is, we should prove that for any $J \subseteq\{1, \ldots, k\}$ there is $I \subseteq\{1, \ldots, k\}$ with $I \neq J$ such that $\sum_{s \in I} \omega_{s} / \beta_{s}-\sum_{s \in J} \omega_{s} / \beta_{s} \in O_{K}$.

Let $\left\{1, \gamma, \ldots, \gamma^{n-1}\right\}$ be a power integral basis of $K$, and define $\psi$ as in Lemma 3.1.

Let $x_{0}, \ldots, x_{n-1} \in \mathbb{Z}$ and $x=\sum_{r=0}^{n-1} x_{r} \gamma^{r}$. Since $-x \in O_{K}$ is covered by $\mathcal{A}=\left\{\alpha_{s}+\beta_{s} O_{K}\right\}_{s=1}^{k}$, we have

$$
\begin{aligned}
0 & =\prod_{s=1}^{k}\left(1-e^{2 \pi i \psi\left(\omega_{s}\left(x+\alpha_{s}\right) / \beta_{s}\right)}\right)=\sum_{I \subseteq\{1, \ldots, k\}}(-1)^{|I|} \prod_{s \in I} e^{2 \pi i \psi\left(\omega_{s}\left(x+\alpha_{s}\right) / \beta_{s}\right)} \\
& =\sum_{I \subseteq\{1, \ldots, k\}}(-1)^{|I|} \prod_{s \in I} e^{2 \pi i\left(\psi\left(\omega_{s} \alpha_{s} / \beta_{s}\right)+\sum_{r=0}^{n-1} x_{r} \psi\left(\omega_{s} \gamma^{r} / \beta_{s}\right)\right)} \\
& =\sum_{I \subseteq\{1, \ldots, k\}}(-1)^{|I|} e^{2 \pi i \psi\left(\sum_{s \in I} \omega_{s} \alpha_{s} / \beta_{s}\right)} \prod_{r=0}^{n-1} e^{2 \pi i x_{r} \psi\left(\sum_{s \in I} \omega_{s} \gamma^{r} / \beta_{s}\right)} \\
& =\sum_{\theta_{0} \in S_{0}} e^{2 \pi i x_{0} \theta_{0}} \sum_{\theta_{1} \in S_{1}} e^{2 \pi i x_{1} \theta_{1}} \ldots \sum_{\theta_{n-1} \in S_{n-1}} e^{2 \pi i x_{n-1} \theta_{n-1}} f\left(\theta_{0}, \ldots, \theta_{n-1}\right),
\end{aligned}
$$


where

$$
S_{r}=\left\{\left\{\psi\left(\sum_{s \in I} \frac{\omega_{s} \gamma^{r}}{\beta_{s}}\right)\right\}: I \subseteq\{1, \ldots, k\}\right\}
$$

and

$$
f\left(\theta_{0}, \ldots, \theta_{n-1}\right)=\sum_{\substack{I \subseteq\{1, \ldots, k\} \\\left\{\psi\left(\sum_{s \in I} \omega_{s} \gamma^{r} / \beta_{s}\right)\right\}=\theta_{r} \\ \text { for all } r=0, \ldots, n-1}}(-1)^{|I|} e^{2 \pi i \psi\left(\sum_{s \in I} \omega_{s} \alpha_{s} / \beta_{s}\right)} .
$$

For each $r=0, \ldots, n-1$, if $\sum_{\theta_{r} \in S_{r}} e^{2 \pi i x_{r} \theta_{r}} F\left(\theta_{r}\right)=0$ for all $x_{r}=0, \ldots$, $\left|S_{r}\right|-1$, then $F\left(\theta_{r}\right)=0$ for every $\theta_{r} \in S_{r}$, because the Vandermonde determinant $\operatorname{det}\left(e^{2 \pi i x_{r} \theta_{r}}\right)_{0 \leqslant x_{r}<\left|S_{r}\right|, \theta_{r} \in S_{r}}$ does not vanish. So, by the above, we have $f\left(\theta_{0}, \ldots, \theta_{n-1}\right)=0$ for all $\theta_{0} \in S_{0}, \ldots, \theta_{n-1} \in S_{n-1}$.

Now suppose that $\mu \in K$ and $\sum_{s \in J} \omega_{s} / \beta_{s} \in \mu+O_{K}$ for a unique subset $J$ of $\{1, \ldots, k\}$. We want to deduce a contradiction.

Set $\theta_{r}=\left\{\psi\left(\mu \gamma^{r}\right)\right\}$ for $r=0, \ldots, n-1$. For any $I \subseteq\{1, \ldots, k\}$ we have

$$
\begin{aligned}
& \left\{\psi\left(\sum_{s \in I} \frac{\omega_{s} \gamma^{r}}{\beta_{s}}\right)\right\}=\theta_{r} \text { for all } r=0, \ldots, n-1 \\
\Longleftrightarrow & \psi\left(\left(\sum_{s \in I} \frac{\omega_{s}}{\beta_{s}}-\mu\right) \gamma^{r}\right) \in \mathbb{Z} \text { for all } r=0, \ldots, n-1 \\
\Longleftrightarrow & \left.\sum_{s \in I} \frac{\omega_{s}}{\beta_{s}} \in \mu+O_{K} \quad \text { (by Lemma } 3.1\right) \\
\Longleftrightarrow & I=J .
\end{aligned}
$$

Thus the expression of $f\left(\theta_{0}, \ldots, \theta_{n-1}\right)$ only contains one summand, and therefore

$$
0=f\left(\theta_{0}, \ldots, \theta_{n-1}\right)=(-1)^{|J|} e^{2 \pi i \psi\left(\sum_{s \in J} \omega_{s} \alpha_{s} / \beta_{s}\right)} \neq 0
$$

which is a contradiction.

The proof of Theorem 1.2 is now complete.

\section{ACKNOWLEDGMENT}

The authors are indebted to the referee for his/her valuable suggestions.

\section{REFERENCES}

[E97] P. Erdős, Some of my favorite problems and results, in: The Mathematics of Paul Erdős, I, 47-67, Algorithms Combin., 13, Springer, Berlin, 1997. MR1425174 (98e:11002)

[G04] R. K. Guy, Unsolved Problems in Number Theory, 3rd edition, Springer, New York, 2004. MR2076335 (2005h:11003)

[J68] J. H. Jordan, A covering class of residues with odd moduli, Acta Arith. 13 (1968), 335-338. MR0220657 (36:3709)

[PS] Š. Porubský and J. Schönheim, Covering systems of Paul Erdös: Past, present and future, in: Paul Erdös and his Mathematics. I (edited by G. Halász, L. Lovász, M. Simonvits, V. T. Sós), Bolyai Soc. Math. Studies 11, Budapest, 2002, pp. 581-627. MR1954716 (2004d:11006)

[S95] Z. W. Sun, Covering the integers by arithmetic sequences, Acta Arith. 72 (1995), 109-129. MR1347259(96k:11013)

[S96] Z. W. Sun, Covering the integers by arithmetic sequences. II, Trans. Amer. Math. Soc. 348 (1996), 4279-4320. MR:1360231 (97c:11011)

[S97] Z. W. Sun, Exact $m$-covers and the linear form $\sum_{s=1}^{k} x_{s} / n_{s}$, Acta Arith. 81 (1997), 175198. MR 1456240 (98h:11019) 
[S99] Z. W. Sun, On covering multiplicity, Proc. Amer. Math. Soc. 127 (1999), 1293-1300. MR.1486752 (99h:11012)

[S03] Z. W. Sun, Unification of zero-sum problems, subset sums and covers of $\mathbb{Z}$, Electron. Res. Announc. Amer. Math. Soc. 9 (2003), 51-60. MR1988872 (2004i:11017)

[S05] Z. W. Sun, On the range of a covering function, J. Number Theory 111 (2005), 190-196. MR2124049 (2005m:11015)

[S07] Z. W. Sun, A connection between covers of the integers and unit fractions, Adv. in Appl. Math., 38 (2007), 267-274.

[Z89] M. Z. Zhang, A note on covering systems of residue classes, Sichuan Daxue Xuebao (Nat. Sci. Ed.) 26 (1989), Special Issue, 185-188. MR1059702 (92c:11003)

Department of Mathematics, Nanjing University, Nanjing 210093, People's Republic OF CHINA

E-mail address: haopan79@yahoo.com.cn

Department of Mathematics, Nanjing University, Nanjing 210093, People's Republic OF CHINA

E-mail address: zwsun@nju.edu.cn 